\title{
Animal models of diabetes-associated vascular diseases: An update on available models and experimental analysis
}

\author{
Judy Choi $^{1}$, Judy de $\operatorname{Haan}^{1}$, and Arpeeta Sharma ${ }^{1}$ \\ ${ }^{1}$ Baker Heart and Diabetes Institute
}

November 27, 2020

\begin{abstract}
Diabetes is a chronic metabolic disorder associated with the accelerated development of macrovascular (atherosclerosis, coronary artery disease) and microvascular complications (nephropathy, retinopathy and neuropathy), which remain the principal cause of mortality and morbidity in this population. Current understanding of cellular and molecular pathways of diabetes-driven vascular complications as well as therapeutic interventions have arisen from studying disease pathogenesis in animal models. Diabetes-associated vascular complications are multi-faceted, involving the interaction between various cellular and molecular pathways. Thus, the choice of an appropriate animal model to study vascular pathogenesis is important in our quest to identify innovative and mechanism-based targeted therapies to reduce the burden of diabetic complications. Herein, we provide up-todate information on available mouse models of both Type 1 and Type 2 diabetic vascular complications as well as experimental analysis and research outputs.
\end{abstract}

\section{Animal models of diabetes-associated vascular diseases:}

\section{An update on available models and experimental analysis}

Judy S.Y. Choi ${ }^{1}$, and Judy B. de Haan ${ }^{1,3,4,5^{*}}$ and Arpeeta Sharma ${ }^{1,2^{*}}$

${ }^{1}$ Baker Heart and Diabetes Institute, Melbourne, Victoria, Australia

${ }^{2}$ Department of Diabetes, Monash University, Central Clinical School, Melbourne, Victoria, Australia

${ }^{3}$ Dept Immunology and Pathology, Central Clinical School, Monash University, Melbourne, Vic, Australia

${ }^{4}$ Department of Physiology, Anatomy and Microbiology, La Trobe University, Melbourne, Australia

${ }^{5}$ Faculty of Science, Engineering and Technology, Swinburne University, Melbourne Australia.

*Joint Senior authors

Addresses for correspondence:

\section{Dr. Arpeeta Sharma}

Group Leader (Oxidative Stress Laboratory)

Diabetic Complications Division

Baker Heart and Diabetes Institute

75 Commercial Road

Melbourne, Victoria 3004, Australia

Phone : +61(3) 85321179 
Fax : +61(3) 85321100

e-mail : Arpeeta.sharma@baker.edu.au

\title{
DECLARATIONS
}

\section{Availability of data and materials}

Non-applicable

\section{Funding}

AS is supported by an early career fellowship from the National Health and Medical Research Council (NHMRC) and a Diabetes Australia Research Project (DARP) General Grant. JBDH is supported by a Baker Fellowship. This work was also supported by operational infrastructure grants through the Australian Government Independent Research Institute Infrastructure Support Scheme (9000220) and the Victorian State Government Operational Infrastructure Support, Australia.

\section{Competing interests}

The authors declare that they have no competing interests.

\section{Ethics}

Ethics approval was not required for the current submission

\section{Author Contributions}

AS and JBDH conceived and designed the review. JC, JBDH and AS provided intellectual input, wrote and edited the review. JC and AS prepared the figures. JC, JBDH and AS approved final version of the manuscript.

A/Prof Judy de Haan and Dr Arpeeta Sharma take full responsibility for the work as a whole, and the decision to submit and publish the review.

\begin{abstract}
Diabetes is a chronic metabolic disorder associated with the accelerated development of macrovascular (atherosclerosis, coronary artery disease) and microvascular complications (nephropathy, retinopathy and neuropathy), which remain the principal cause of mortality and morbidity in this population. Current understanding of cellular and molecular pathways of diabetes-driven vascular complications as well as therapeutic interventions have arisen from studying disease pathogenesis in animal models. Diabetes-associated vascular complications are multi-faceted, involving the interaction between various cellular and molecular pathways. Thus, the choice of an appropriate animal model to study vascular pathogenesis is important in our quest to identify innovative and mechanism-based targeted therapies to reduce the burden of diabetic complications. Herein, we provide up-to-date information on available mouse models of both Type 1 and Type 2 diabetic vascular complications as well as experimental analysis and research outputs.
\end{abstract}

\section{Introduction}

Diabetes Mellitus (DM), either Type1 (insulin-deficient) or Type2 (adult onset, insulin resistance), is a chronic metabolic disorder that has serious implications on cardiovascular health. Indeed, macro- and microvascular complications associated with diabetes account for approximately $65 \%$ of deaths in the diabetic population $[1,2]$. Macrovascular complications associated with diabetes are mainly represented by atherosclerosis and/or hypertension and their sequelae, which include heart attacks and stroke, whilst microvascular complications include chronic kidney disease, retinopathy and neuropathy. The increased risk of cardiovascular complications in diabetes is attributed to the elevated blood glucose levels and the interplay between different cellular mechanisms in the disease setting as a consequence of hyperglycemia[3]. These include, but are not limited to, genetic, epigenetic, and cellular signalling pathways, particularly the interplay between 
oxidative stress and inflammation, which then result in imbalances in cellular metabolism contributing to the development of cardiovascular complications.

Current standard of care for diabetic patients focusses on glucose-lowering, lipid lowering and blood pressure control, however these treatment options have yielded suboptimal outcomes on cardiovascular end points, with the development of tolerance and resistance to these therapies, particularly with respect to blood pressure lowing medications limiting their long-term use $[4,5]$. Newer, more innovative CV-specific drug treatments are urgently needed to prevent diabetes-mediated vascular diseases. In this regard, animal models have proven to be an invaluable tool in exploring and characterising novel pathophysiological pathways, identifying therapeutic targets and evaluating their in vivo potential. However, it is critical to choose appropriate animal models that most closely mimic the human pathophysiology of diabetes and its sequalae such as the cardiovascular complications, to investigate potential treatments and therapeutic strategies. To date, there is no perfect animal model that encompasses all the pathophysiology and characteristics of human disease but with advances in genetic engineering and chemical manipulation, rodents have proven to be the most reproducible, effective and preferred animal model for studies of diabetic vascular diseases, particularly with respect to emulating specific conditions that occur in humans. In this review, we provide a comprehensive and up-to-date analysis on proven animal models of diabetes-associated vascular diseases, with a focus on the analysis and experimental outputs that can be achieved with these models.

\section{Animal models of diabetes-associated macrovascular diseases}

\section{Vascular pathophysiology: key features that need to be represented in animal models}

Atherosclerosis is the principal cause of death and disability in the diabetic population, with the incidence of an atherosclerotic event occurring approximately 14.6 years earlier in this patient cohort. The pathophysiological events that lead to disease progression in the presence of diabetes have been extensively reviewed[1, $6,7]$, however, key factors that are important to consider in choosing a relevant animal model of diabetesassociated atherosclerosis will be discussed. A main clinical feature that presents in uncontrolled or poorly controlled Type1 and Type2 diabetic patients is hyperglycemia, the key driver of vascular injury. Elevated blood glucose, primarily via advanced glycation end products (AGEs) and their interaction with receptor for advanced glycated end products (RAGE) drives pro-inflammatory/pro-oxidant pathways to elicit molecular, cellular and vascular injury[8, 9]. A further key feature of diabetes is dyslipidemia manifested through elevated triglycerides, decreased high density lipoproteins (HDL) and changes in low density lipoprotein (LDL) structure to a more atherogenic profile[10], which results from the ineffective clearance of post-prandial lipoprotein rather than increased circulating levels of fasting lipoproteins[11]. In addition, the heightened state of oxidative stress and inflammation perturbs cellular metabolism particularly within endothelial cells which leads to endothelial dysfunction. Together with a build-up of oxidised LDL particles, endothelial dysfunction promotes increased adhesion and infiltration of leukocytes into the vessel wall and the formation of foam cells, thereby leading to the acceleration of atherosclerotic processes[1].

Diabetes-associated atherosclerosis is most commonly studied in mice despite the fact that humans and mice differ in certain characteristics that affect the development of disease progression. Firstly, mice are generally protected from atherogenesis due to differences in their lipoprotein profile, with mice exhibiting elevated HDL levels due to the absence of cholesteryl ester transfer protein (CETP) [12, 13]. Therefore, atherosclerotic plaque generation requires genetic manipulation such as the deletion of the apolipoprotein $\mathrm{E}$ (ApoE) gene or the LDL receptor (LDLR) gene together with dietary interventions. Additionally, humans and mice have differences in cardiovascular anatomy and haemodynamic physiological forces that influence the sites of atherosclerosis[14]. Lesions predominantly occur in the coronary arteries, carotid and peripheral vessels in humans whilst in mice, lesions are commonly studied in the aortic sinus and the aortic arch[14]. A summary of the models of diabetes-associated atherosclerosis is shown in Figure 1.

Rodent models of diabetes-associated atherosclerosis2.2.1. Models of Type1 diabetes-associated atherosclerosis

To replicate human disease, most mouse models of Type1 diabetes are insulin deficient, either by chemical induction or genetic manipulation. The most extensively studied Type1 model of diabetes-associated 
atherosclerosis is the streptozotocin (STZ) induction model in the ApoE knockout (-/-) mouse, initially characterised by Park et al[15]. A major advantage of the STZ-induced ApoE $\mathrm{E}^{-/-}$mouse model is the vast amount of published literature available utilising this model allowing researchers to compare and contrast data, cellular mechanisms and therapeutic interventions. STZ, a pancreatic $\beta$-islet cell toxin, enters the cell via Glut-2 transporters to mediate its cytotoxic effect, thereby limiting insulin production, leading to hyperglycaemia[16]. Generally, STZ is administered as five consecutive low doses (55mg/kg/day)[17-19] but certain groups have demonstrated more consistent elevations in blood glucose by using two consecutive high doses $(100 \mathrm{mg} / \mathrm{kg} /$ day)[20-24]. This becomes particularly important for longer term studies where blood glucose levels may decline due to recovery of $\beta$-islet cells. Despite the benefits of a robust and consistent increase in hyperglycemia of around $25-30 \mathrm{mmol} / \mathrm{L}$, there are limitations that may preclude the use of STZ under certain circumstances. For example, STZ at high doses has been shown to affect the function of other cells expressing the Glut-2 transporter, such as hepatocytes and epithelial cells of the renal tubules, which may be exposed to the toxic action of STZ leading to unwanted side effects in the liver and kidneys[25]. In addition, a further limitation of using STZ as the chemical inducer of diabetes is that females, particularly mice, have been shown to be resistant to STZ, often requiring higher doses of STZ to obtain equivalent levels of hyperglycemia compared to male counterparts[26]. Thus, female mice are commonly omitting in preclinical research for diabetic complications when STZ is the preferred method of Type1 diabetes induction. STZ also exhibits broad spectrum antibacterial properties which may alter the gut microbiota[27].

Despite the noted limitations, the STZ-induced diabetic Apo: ${ }^{-/}$mouse model is the preferred model for studying atherosclerosis, since these mice exhibit hyperglycemia as well as dyslipidemia, as indicated by the elevated blood glucose levels, total cholesterol, LDL and triglyceride levels. Plaque formation is increased 3-6 fold in this model over a 10-20 week period, with early discrete lesions observed within the aortic sinus and the aortic arch; however, over a longer course of diabetes, lesions additionally develop over the thoracic and abdominal aortic regions. This model has been associated with endothelial dysfunction, increased inflammation and oxidative stress as well as macrophage foam cell formation, all of which are hallmarks of atherosclerotic pathogenesis. More importantly, the STZ-induced ApoE ${ }^{-/-}$mouse model has been particularly useful in investigating potential therapies, such as but not limited to soluble RAGE (sRAGE), peroxisome proliferator-activator gamma agonists, anti-oxidants (ebselen, dimethyl furate), SGLT2 inhibitors and ApoA1 mimetics, in their ability to attenuate diabetes-associated atherosclerosis, independent of blood glucose lowering effects $[21,22,28-32]$. As the $\mathrm{ApoE}^{-/-}$mouse model is well established and easy to breed, cross-breeding ApoE $/$ - mice with mice harbouring genetically altered proteins such as transcription factors, receptors and/or enzymes enables the elucidation of their role in diabetic atherosclerotic mechanisms. On the contrary, STZ-induced diabetic LDLR ${ }^{-/}$mice do not exhibit extensive atherosclerotic lesions despite having hyperglycemia and elevated LDL/vLDL levels and often require additional diet supplementation[33] making them a more ideal model of Type2 diabetes.

A less well-known model that is used in Type1 diabetic atherosclerotic research, particularly if the side-effects of STZ are of concern (as detailed above), is the spontaneous genetic Akita mouse, cross bred with either the $\mathrm{ApoE}^{-/-}$or LDLR $\mathrm{LL}^{-/-}$mouse. The Akita mouse, first selected in Japan, carries an autosomal dominant missense mutation in the insulin2 gene, resulting in impaired proinsulin processing and aggregation of misfolded insulin protein, rendering it insulin deficient. Akita mice develop typical Type1 diabetes characteristics, including hyperglycemia, polyuria and polydipsia, as well as impaired vascular tone as early as 3-4 weeks of age, making it an ideal model to study the effects of diabetes on endothelial dysfunction and macrovascular complications[34]. When cross-bred to the $\mathrm{ApoE}^{-/-}$or $\mathrm{LDLR}^{-/}$mouse, this model exhibits dyslipidemia, mainly manisfested as increases in triglycleride and non-HDL cholesterol levels, and an approximately 2 to 3 -fold increase in atherosclerotic lesions[35-37]. This is accompanied by increased infiltration of immune cells into the plaque, in particular macrophages and T-cells, as well as augmentation of vascular inflammation as demonstrated by increased levels of TNF- $\alpha$, IL- $1 \beta$ and MCP-1, all of which are key mediators involved in plaque progression[36, 37]. Interestingly, the differences in plaque size are not as pronounced in female Akita/LDLR ${ }^{-/-}$mice as they are in male mice, suggesting gender differences could play a part in diabetic atherosclerosis in this model. 
The models described thus far are promising to investigate the mechanisms of endothelial dysfunction, vascular inflammation and plaque formation in a diabetic setting, however, it is difficult to discern whether these arise as a direct effect of the hyperglycemia or as a consequence of other confounding atherogenic factors such as the dyslipidemia under these conditions. To circumvent this problem, Vikramadithyan et al have developed a mouse model to study the effects of hyperglycemia in accelerating atherogenesis by manipulating Aldose reductase, an enzyme involved in catalysing the reduction of glucose to sorbitol, in combination with STZ[38]. Aldose reductase has been shown to accelerate diabetic vascular complications through the increased production of reactive oxygen species (ROS). Indeed, overexpression of human aldose reductase in the diabetic $\mathrm{LDLR}^{-/-}$mouse resulted in a 2-fold increase in lesion size as compared to diabetic LDLR $^{-/-}$mice alone, driven by defects in glucose-driven ROS signalling[38]. Another model developed to closely mimic the autoimmune destruction of pancreatic $\beta$ cells as observed in human Type1 diabetes is the induction of lymphocytic choriomeningitis virus (LCMV) infection [39, 40]. The LCMV glycoprotein (GP) transgene is controlled by the insulin promoter in $\beta$ cells, which is rapidly destroyed upon infection. These $\mathrm{LDLR}^{-/}$mouse injected with the LCMV GP developed severe hyperglycemia that was associated with accelerated lesion formation in the absence of hypertriglyceridemia[39, 40]. Moreover, atherosclerotic lesions were morphologically similar to human lesions particularly with increased arterial macrophage accumulation and intralesional haemorrhage[39]. Nonetheless, further investigation is required to delineate the exact role of altered lipoprotein profiles and hyperglycemia in preclinical animal models of Type1 diabetes-induced atherosclerosis.

\subsubsection{Models of Type2 induced diabetes-associated atherosclerosis}

The prevalence of Type 2 diabetes driven by insulin resistance and obesity is on an exponential rise worldwide with 700 million individuals predicted to have Type 2 diabetes by 2045[41]. Metabolic features of Type2 diabetes include hyperglycemia, hyperinsulinemia, obesity and dyslipidemia encompassing an increase in LDL, vLDL and a decrease in HDL levels. Thus, it is pertinent to choose an animal model that at best encompasses all of these features in order to study the mechanisms and explore therapeutic approaches to limit macrovascular disease induced by Type 2 diabetes. The majority of the animal models available to study Type2 diabetes-associated atherosclerosis are either genetically manipulated or diet induced.

Two of the most common genetic models are generated by crossbreeding genetic models of Type 2 diabetes, such as the leptin (ob/ob) and leptin receptor $(\mathrm{db} / \mathrm{db})$ deficient mice, with atherosclerotic mice such as the $\mathrm{ApoE}^{-/-}$and $\mathrm{LDLR}^{-/-}$mice. Leptin, the satiety hormone that is produced by adipose cells and enterocytes of the small intestine, regulates appetite via receptors in the arcuate nucleus of the hypothalamus. Ob/ob mice and $\mathrm{db} / \mathrm{db}$ mice carry mutations in the leptin gene and the leptin receptor-coding gene respectively, causing the induction of hyperphagia leading to obesity and Type 2 diabetes[42, 43]. The db/db;ApoE $E^{-/}$ mouse is well established and exhibits typical features of Type 2 diabetes by 20 weeks, such as increased body weight, hyperglycemia, hyperinsulinemia, elevated cholesterol levels (triglycerides, LDL and vLDL) with a 3-4 fold increase in atherosclerotic plaque burden[42, 43]. From a more diabetic perspective, these mice display higher expression of RAGE, inflammatory adhesion molecules (such as VCAM-1) and matrix metalloproteinase (MMP-9) activity[43]. Pharmacological interventions, such as soluble RAGE and PPARagonists have proven to be effective in their pre-clinical evaluation with this model[43]. Similar atherosclerotic observations were noted in the ob/ob;ApoE ${ }^{-/-}$and ob/ob;LDLR ${ }^{-/}$mice, however, in these models either hyperglycemia was not evident (ob/ob; $\mathrm{ApoE}^{-/-}$mice) or hyperlipidemia was too pronounced masking the diabetic effects (ob/ob;LDLR ${ }^{-/-}$mice). A modification of the ob/ob;ApoE ${ }^{-/-}$and ob/ob;LDLR ${ }^{-/}$mice to more closely align with the human lipoprotein profile is to cross these mice to $\mathrm{ApoB}^{100 / 100}$ producing mice which do not make ApoB-48 in their liver[44, 45]. Cholesterol in mice is mostly carried in ApoB-48 containing vLDL particles and chylomicron remnants whereas humans do not produce ApoB-48 in the liver[44, 45]. $\mathrm{Ob} / \mathrm{ob} ; \mathrm{ApoE}^{-/-} ; \mathrm{ApoB}^{100 / 100}$ and ob/ob;LDLR ${ }^{-/-} ; \mathrm{ApoB}^{100 / 100}$ mice exhibit a metabolic phenotype which includes obesity, insulin resistance as well as atherosclerosis and hypertension[44]. Due to their specificity in lipoprotein profiles, these models are useful for studying cholesterol-lowering therapies from a Type2 diabetic/metabolic syndrome perspective[44]. 
For research that requires less influence of lipid levels and consistent features of Type2 diabetes, there are other models with a more clinically relevant lipoprotein profile that can be considered. The $\mathrm{LDLR}^{-/-}$mouse that is able to synthesise only $\mathrm{ApoB}^{100 / 100}$ protein represents a model more closely aligned to the human lipoprotein profile. This model exhibits accelerated atherogenesis[45]. Furthermore, Heinonen et al crossbred the $\mathrm{LDLR}^{-/-} ; \mathrm{ApoB}^{100 / 100}$ mouse with transgenic mice overexpressing insulin-like growth factor-II in pancreatic $\beta$ cells which led to the development of Type 2 diabetes without major hyperlipidemia[46]. These mice demonstrated aggravated atherosclerotic lesions with increased complications such as lesion calcification which coincided with an increase in the expression of a variety of genes involved in calcification (OPN, ALP-2 and BMP-2) and inflammation (MCP-1)[46]. Moreover, the complexity of the lesion and increased calcification was more prominent in aged mice, which is in line with clinical studies that demonstrate calcification in peripheral and coronary arteries, particularly in older patients with a longer duration of diabetes, and is considered a strong independent risk factor for cardiovascular events[46-49]. Another useful genetic model is the $\mathrm{ApoE}^{-/-}$mouse that has a homozygous or heterozygous deletion of the insulin receptor substrate (IRS1 and IRS2) gene. ApoE ${ }^{-/-}$mice that are IRS1 ${ }^{+/-}, \mathrm{IRS}^{+/-}$and IRS2 $2^{-/-}$mice display a Type2 diabetes/metabolic syndrome phenotype that includes, insulin resistance, hyperglycemia, hyperinsulinemia, and impaired glucose tolerance as compared to IRS2 $2^{+/+}$mice, however lipid levels were only modestly altered[50-52]. This is consistent with clinical findings in Type2 diabetic patients that have reduced expression of IRS2 in their pancreatic $\beta$ cells and a highly polymorphic IRS1 gene[53]. These mice have augmented atherosclerotic lesions and vascular inflammation that is attributed to impaired IRS2 signalling of the AKT and ERK pathways that lead to the upregulation of pro-atherogenic MCP-1[54]. Lastly, another note-worthy genetic model of T2D-induced atherosclerosis is the heterozygous glucokinase (GK) knockout mouse on the ApoE $/-$ background. GK is the rate-limiting enzyme of glucose-stimulated insulin secretion, thus is it not surprising that $\mathrm{GK}^{+/-} / \mathrm{ApoE}^{-/-}$mice on a western diet show significant glucose intolerance and impaired glucose-stimulated insulin secretion, a phenomenon that was consistent over time[55]. Despite plasma lipid levels being comparable to the $\mathrm{ApoE}^{-/-}$mouse, $\mathrm{GK}^{+/-} / \mathrm{ApoE}^{-/-}$mice have significantly accelerated and highly developed atherosclerotic lesions, strongly suggesting that lesions are driven by hyperglycemia and insulin resistance[55].

Diet-induced insulin resistance is extensively studied in models of Type2 diabetes associated atherosclerosis. In particular, a high-fat western diet (comprising cholesterol $(0.2 \%$ total cholesterol) + total fat $(21 \%$ by weight; $42 \%$ kcal from fat) + high saturated fatty acids ( $>60 \%$ of total fatty acids) + high sucrose $(34 \%$ by weight)) in $\mathrm{ApoE}^{-/-}$or $\mathrm{LDLR}^{-/-}$mice increases lesions throughout the aortic tree and exhibits hyperglycemia, hyperinsulinemia and hypertriglyceridemia. Interestingly, in mice fed a diabetogenic diet where $35 \%$ of the energy is sourced from fats, atherosclerotic lesions were inconsistent with the LDLR ${ }^{-/}$mice being more susceptible to diabetes[56, 57]. $\mathrm{LDLR}^{-/}$mice on a diabetogenic diet had severe dyslipidemia and increased atherosclerotic lesions, albeit with modest increases in glucose and insulin levels. On the contrary, diabetogenic diet-fed $\mathrm{ApoE}^{-/-}$mice displayed no significant differences between in lipids, glucose and atherosclerotic lesion sizes as compared to chow-fed $\mathrm{ApoE^{-/- }}$ mice[56, 57]. In comparing neointimal formation, $\mathrm{ApoE}^{-/-}$mice fed a western diet exhibited larger lesions, accompanied by higher glucose and insulin levels, as compared to mice on a diabetogenic diet that maintained euglycemia but developed insulin resistance[58]. Treatment of these mice with Rosiglitazone, a thiazolidinedione class of PPAR agonist, led to a marked reduction in macrophage content and neointimal formation[58].

Due to ongoing discrepancies in lesion size between diet-induced and genetically manipulated mouse models, research groups have continually strived to modify diet composition to more closely reflect the human Type 2 diabetic cardio-metabolic risk profile. Indeed, a diabetogenic diet supplemented with $0.15 \%$ cholesterol in the LDLR $^{-/-}$mice further enhances obesity, insulin resistance, systemic inflammation and atherosclerosis with increased macrophage accumulation[59, 60]. Additionally, Neuhofer et al found that by altering complex carbohydrates with a high-fat, high-sucrose diet and the inclusion of $0.15 \%$ cholesterol, they were able to further augment atherosclerotic lesion size and increase adipose tissue inflammation, hallmarks often seen in human obesity and insulin resistance, thus creating a more robust model of metabolic syndrome with dietinduced obesity[61]. Furthermore, supplementing rodent diets with high fructose to emulate consumption of 
sugary drinks in humans and the impact on atherosclerotic lesions has been investigated by several groups. $\mathrm{ApoE}^{-/}$mice fed a high fructose diet exhibited a pro-atherogenic state with insulin resistance, independent of hypercholesterolemia[62]. This cohort of mice exhibited increased vascular inflammation (VCAM-1), overproduction of NADPH-oxidase induced ROS and significant outward vascular remodelling[62]. Supplementation of liquid fructose to western-diet fed $\mathrm{LDLR}^{-/}$mice demonstrated similar results with the mice developing increased atheromatous plaque driven by increased monocyte/macrophage infiltration and local inflammation. Importantly, atherosclerotic plaque size strongly correlated with plasma lipid levels[63]. Another method that has been described to model the metabolic syndrome associated with diabetes is the incorporation of low-dose STZ along with high fat feeding. Although this model has not been validated in terms of atherosclerotic lesions, it has been shown to develop cardiac inflammation and dysfunction, which combines both early and late stages of the disease[64, 65].

\subsubsection{Models of atherosclerotic plaque complications}

In advanced stages of lesion development, the atherosclerotic plaque is more vulnerable, unstable and prone to rupture. Indeed, plaque rupture frequently leads to luminal thrombosis which is the direct cause of acute ischaemia responsible for cardiovascular deaths and this phenomenon is accelerated in the diabetic setting $[66$, 67]. However, plaque rupture is rarely detected in mouse models of atherosclerosis, a limitation with respect to replication of human pathology to recapitulate the human disease. Nonetheless, several experimental models have proven advantageous in analysing post-rupture thrombotic events. In general, to induce unstable plaques, an invasive approach such as the placement of a perivascular carotid cuff or ligation of the common carotid artery under western diet-fed conditions in the ApoE $\mathrm{E}^{-/-}$mouse can achieve an environment of altered haemodynamic flow to replicate the advanced stages of atherosclerotic plaque development with evidence of an occlusive thrombus[68]. Another method commonly used to develop the unstable plaque phenotype is through the elevation of Angiotensin II, either endogenously via surgical clipping of the renal vasculature, or via Angiotensin II infusion through the placement of osmotic mini pumps. High angiotensin II levels in the western-diet fed $\mathrm{ApoE}^{-/-}$mice exhibit accelerated lesions with characteristic features of a thinner fibrous cap, larger lipid necrotic core, and increased macrophage content, all of which contribute to plaque destabilisation and vulnerability $[69,70]$. Another interesting model that displays features of advanced unstable atherosclerotic plaque is a model harbouring a heterozygous mutation in the fibrillin- 1 gene $\left(\mathrm{C} 1039^{+/-}\right)$which leads to the fragmentation of elastic fibres in the vessel wall, increased arterial wall stiffness and highly sporadic plaque rupture when crossed to the $A p o E^{-/-[71,72]}$. When fed a western diet for 35 weeks, ApoE ${ }^{-/} \mathrm{Fbn} 1^{\mathrm{C} 1039 \mathrm{G}+/-}$ mice exhibit a 3 -fold increase in necrotic core, which was associated with increased T-cell infiltration, augmented neovascularization and intraplaque haemorrhage[72]. Furthermore, spontaneous plaque rupture was observed in the ascending aorta and brachiocephalic arteries in more than $50 \%$ of $\mathrm{ApoE}^{-/-} \mathrm{Fbn1}{ }^{\mathrm{C} 1039 \mathrm{G}+/-}$ mice[72]. In line with human complications, these $\mathrm{ApoE} \mathrm{E}^{-/-} \mathrm{Fbn} 1{ }^{\mathrm{C} 1039 \mathrm{G}+/-}$ mice showed coronary plaques and myocardial infarction as well as sudden death[72]. Thus, this reproducible model of advanced atherosclerosis and plaque rupture with added complications of myocardial infarction is clinically relevant.

While these models are useful in studying the pathways involved in plaque rupture, they are not entirely ideal as they are generated by an artificial environment often driven by genetic manipulation or altered biomechanical stress. Therefore, there is a need to develop preclinical models that represent the spontaneous plaque rupture that occurs in humans. A more innovative approach has been developed by Chen et al who showcase a unique mouse model of atherosclerotic plaque instability that is proving to be useful for mechanistic insights and advantageous for therapeutic testing[73]. With the hypothesis that low shear stress and high tensile stress contribute to plaque instability in patients, computational haemodynamic modelling was used to develop a unique tandem stenosis mouse model of plaque instability/rupture. The tandem stenosis model involves applying two sutures to the right common carotid artery in the ApoE ${ }^{-/-}$mouse fed a high-fat western diet, resulting in an unstable plaque reflecting the characteristics seen in humans. These features include ruptured fibrous caps, intraplaque haemorrhage, large necrotic cores, plaque inflammation, intravascular occlusive thrombus formation and near-infrared autofluorescence. Importantly, this model of plaque instability and rupture is suitable for testing the responsiveness of pharmacological interventions 
including plaque-stabilising drugs and other inflammatory mediators, however, its role in the diabetic setting is yet to be determined.

Experimental analysis of diabetes-associated atherosclerosis

In most preclinical animal models, atherosclerotic lesion size is the first quantitative measure performed to determine if genetic loss or overexpression as well as pharmacological intervention has an impact on diabetesassociated atherosclerosis. Generally, lesions are observed in 1) aortic root/sinus and ascending aorta, 2) aortic tree which spans the arch, thoracic and abdominal regions and 3) the brachiocephalic (innominate) artery. Quantification of lesion area in the aortic sinus and ascending aorta is described in detail by Paigen et al[74] and Daughtery and Whitman[75]. This involves sectioning the whole frozen aortic root spanning approximately $300 \mu \mathrm{m}$ and the ascending aorta $(400 \mu \mathrm{m})$. Acquaintance with the architecture of the sinus region and the three valve leaflets is essential for the accurate sectioning of this region of tissue requiring considerable skill. Staining of selected sections 30um apart is then performed using Oil Red O, a fat soluble dye for the staining of triglycerides and lipids in atherosclerotic plaque. The advantage of obtaining serial sections through the sinus region is that cellular composition of the plaque (collagen, macrophages, smooth muscle cells and necrotic core) can be determined by histological (H\&E, picosirius red, masson's trichrome) and immunohistochemical staining (CD68 antibody to detect macrophages). Additionally, inflammation and oxidative stress markers can be quantified by performing immunohistochemistry and immunofluorescence using specific antibodies [31, 76-79].

A relatively quick and simple method to quantify atherosclerotic lesions on the intimal surface of the aortic tree is by an en facemethod, whereby the formalin-fixed aorta is stained with Sudan IV, a lipid soluble dye, after which the aorta is cut open, pinned flat and imaged. This mode of analysis is normally one dimensional with lesion area being the main assessment, however, the aorta can then be embedded in paraffin for immunohistochemical analysis to determine inflammatory and oxidative stress markers. Furthermore, using a separate cohort of mice, the aorta can be snap frozen for RNA and protein extraction to determine atherogenic proteins and pathways by qRT-PCR and Western Blotting respectively[20, 76, 80, 81]. Similarly, the innominate artery which is connected to the aortic arch can be dissected out and sectioned to assess lesion area using histological stains. Lesions in the innominate artery develop the complexities of late stage plaque, characterized by an atrophic media, perivascular inflammation, and thinning of the fibrous cap which in some instances can lead to plaque rupture and intraplaque haemorrhage.

A major limitation of these methods is that the analysis does not provide volumetric three-dimensional assessment of the plaque, nor the degree of vascular occlusion. Often tissue integrity is compromised during preparation and processing for histology. To overcome these issues, over the past decade, there have been improvements in imaging technologies to provide a more in-depth view of plaque complexity. For example, an ex vivo scanning method, known as microscopic computed tomography (microCT) has been adapted for the visualisation of atherosclerotic plaque[82]. Excised hearts and vessels from diabetic ApoE-/- mice, can be stained en bloc with metal solutions and scanned using microCT. Then, an image analysis software is able to recreate a virtual histological section to view multi-focal images in both 2-dimensional and 3-dimensional formats, allowing volumetric assessments of the atherosclerotic lesion[82]. The tissue is intact and can further be processed for traditional histology and immunohistochemistry[82]. In addition, ex vivohigh-resolution three dimensional MRI is able to sensitively and volumetrically quantitate atherosclerotic lesions in the mouse aortic root and innominate artery with specific insight into microanatomy of the lipid-rich necrotic core and fibrous cap components which can be verified by histology, allowing for tracking of lesion progression over time[83]. Using fluorescence emission computed technology, Htun et al were able to detect autofluorescence in the near-infrared range that correlated with plaque instability and vulnerability, establishing this technique as a vital imaging tool for the detection of high risk plaques in patients[84].

The small size of mouse aortae can limit the availability of tissue for analysis, often requiring separate cohorts of mice to be run for extensive histological analysis as well as gene expression and protein quantification, making these studies time, labour and cost intensive. To circumvent this, a new emerging technology, known as Laser Capture Microdissection (LCM) is gaining significant interest. This technique can be performed 
on frozen or paraffin-embedded sectioned tissue. LCM enables subpopulations of tissue cells to be collected under the microscope using a low-power infrared laser, which harvests cells of interest directly by first removing unwanted cells to give histologically pure enriched cell populations[85, 86]. Furthermore, this technology preserves the integrity of the tissue and does not damage RNA, DNA and protein, therefore, enabling a number of downstream molecular analyses to be performed. The precision with which tissues are dissected enables the extraction of lesion-specific areas allowing researchers to explore cell populations and differentially expressed genes that make up the different plaque regions. Indeed, LCM of the atherosclerotic lesion showed a significant enrichment of foam-cell specific RNA transcripts[87]. In addition, this cell-specific approach enables analysis of genetic and pharmacological manipulations of atherosclerotic lesions.

Over the last decade, research has focused on examining cell-specific populations and markers that are responsible for disease progression. As such, flow cytometry has proven useful to sort, identify and quantify cell subpopulations within the atherosclerotic aorta. In particular, flow cytometry has been used to track leukocyte infiltration, composition and subtypes in aortae, which is significantly heightened in disease settings[88]. A major advantage of flow-cytometry is the ability to simultaneously stain for surface and intracellular markers using a range of antibodies to identify different cell types and their functional stage[88, 89]. This enables both quantitative and qualitative results from one sample. Using this technique, Nagareddy et al elegantly demonstrated significant increases in pro-inflammatory circulating monocytes (Ly6-C ${ }^{\text {hi }}$ subset) and neutrophils but not lymphocytes, which were significantly lessened after glucose lowering, suggesting a role for leukocytosis in promoting diabetes-associated atherosclerosis[90]. Recently, Brennan et al cultured diseased carotid arteries ex vivo from diabetic and non-diabetic patients who underwent carotid endarterectomies and analysed tissue cytokine responses in response to anti-inflammatory treatment with a pro-resolving mediator known as LipoxinA4[91]. Additionally, other groups have utilised this method to perform flow cytometry of immune cell subsets[92]. This reproducible technique allows the investigation of important aspects of atherogenesis, particularly the function of immune cell and cell-cell interactions within the tissue from a human disease perspective[91, 92].

In line with flow cytometry data, more recently, single-cell RNA sequencing (scRNAseq) technologies allow the evaluation of transcriptome information from a single cell to reveal cell population differences at the RNA level. Moreover, a key feature is the ability to detect heterogeneity among individual cells to define the cellular landscape via cell maps[93]. Since its inception a few years ago, several research groups have employed scRNAseq technology to uncover the transcriptome-based cellular landscape of human and murine atherosclerotic plaque, with a particular focus on unrecognized immune cell populations and their role in atherogenesis[94-97]. Indeed, current work is underway to profile the transcriptional landscape in diabetic atherosclerotic aortae using scRNAseq.

Lastly, as atherosclerosis is a dynamic process involving the interaction between different cell types, it is pertinent to explore the use of real-time functional assays. As an initial step in atherogenesis, vascular inflammation triggers the cell adhesion cascade, contributing to the recruitment and infiltration of leukocytes to the endothelium. Intravital microscopy is now widely used to visualise and record leukocyte rolling and adhesion using whole blood perfusion in real-time[98]. As this method is performed on vessels ex vivo, it does not require invasive surgery in animals. Additionally, this technique has been validated in diabetes-induced vasculopathies enabling localised inhibitor treatment to evaluate the role of therapeutics in modulating leukocyte-endothelial interactions[20, 98, 99]. Vessel myography ex vivo is another important technique to assess endothelial viability and function, which are critical early markers for the development of diabetes-associated atherosclerosis. This technique allows researchers to evaluate various parameters including the assessment of vascular tone, endothelium-dependent and independent function and nitric oxide bioavailability, which can be determined in the presence or absence of pharmacological interventions/genetic manipulations[20, 100]. A summary of experimental and research outputs to evaluate diabetes-associated atherosclerosis is shown in Table 1.

\section{Animal models of diabetes associated microvascular diseases}

In diabetic patients, it is apparent that microvascular and macrovascular complications often develop si- 
multaneously. Microvascular complications are characterised by functional and structural organ damage arising from changes affecting capillaries and arterioles. Three major manifestations of diabetic microvascular disease are nephropathy, retinopathy and neuropathy. While animal models that describe diabetic microvascular diseases have been extensively reviewed[101-103], we aim to highlight the most promising murine models that most closely align with three debilitating human microvascular pathologies, diabetic nephropathy, retinopathy and neuropathy.

Diabetic nephropathy

\subsubsection{Kidney pathophysiology: key features that need to be represented in animal models}

Diabetic nephropathy is a progressive complication of DM, a leading cause of end-stage renal failure globally. A main clinical feature that presents in both Type1 and Type2 diabetic patients is albuminuria where the first manifestation typically occurs as microalbuminuria, defined as albumin excretion of 30-299mg in 24 hours. Over time, microalbuminuria progresses to proteinuria, defined as albumin excretion of over 500mg in 24 hours leading to overt diabetic nephropathy[104]. Hyperglycaemia acts as the major driving force of the progression to diabetic nephropathy with the generation of ROS, formation of AGEs, and the activation of polyol and PKC pathways all contributing to glomerular damage. The downstream consequences include inflammation, thickening of the glomerular basement membrane and microaneurysm formation[105]. In addition, accumulation of extracellular matrix proteins such as collagens and fibronectin replaces healthy renal architecture, leading to mesangial expansion and reduction of filtration surface area, which in turn is further disrupted by the thickening of glomerular basement membranes[106].

\subsubsection{Models of diabetic nephropathy and research outputs}

For many years, mouse models have been the primary and most extensively studied species to investigate kidney pathogenesis and for the development of novel therapies to limit diabetic nephropathy. As previously mentioned, the diabetogenic ß-islet cell toxin, STZ, is also by far the most widely used drug to induce diabetic nephropathy. However, development of overt diabetic nephropathy is strain dependent. C57BL/6J and $\mathrm{BALB} / \mathrm{c}$ strains of mice are highly responsive to developing STZ-mediated diabetes although C57BL/6J mice are relatively resistant to developing diabetic nephropathy[107]. Hence, C57Bl/6J mice are commonly crossbred with genetic mouse models known to have impaired renal function. One approach is to breed eNOS ${ }^{-/-}$ mice onto the C57BL/6 background. Deletion of eNOS diminishes NO availability, thereby phenocopying the well-documented decreased NO levels and its effects on the vasculature in human diabetic nephropathy. STZ-induced diabetic eNOS ${ }^{-/}$mice on the C57BL/6 background develop significant impairment of renal function in which clear pathophysiological changes are visible after 24 weeks of diabetes[108]. On its own, a reduction in eNOS functionality is sufficient to develop vascular dysfunction, hypertension and albuminuria, all of which accelerate renal injury, however, when superimposed on a diabetic background the severity of the albuminuria appears to be enhanced, and more advanced glomerular lesions and glomerular endothelial injury are exhibited in the STZ-induced diabetic eNOS ${ }^{-/}$mouse model. However, high dose administration of STZ should be avoided in this model since this can be highly lethal, although the extent of albuminuria and renal injury observed is milder than that of the $\mathrm{eNOS}^{-/-} \mathrm{db} / \mathrm{db}$ (Type2 diabetes) mouse model[108].

Genetic mouse models, such as the ob/ob and $\mathrm{db} / \mathrm{db}$ mice, have been widely studied as models of diabetic nephropathy, although the degree of severity differs between these two models. In the $\mathrm{db} / \mathrm{db}$ mice, larger glomeruli with increased mesangial matrix are visible by 5 -6month of age, becoming more pronounced over time, along with thickening of the glomerular basement membrane. The extent of renal disease is far less in ob/ob mice when compared to $\mathrm{db} / \mathrm{db}$ mice. When on the $\mathrm{C} 57 \mathrm{Bl} / 6$ background, ob/ob mice are insulin resistant but do not develop severe diabetes. The major renal pathology in ob/ob mice primarily includes diffuse and nodular lipo-hyaline changes in the glomerulus with a relative paucity in mesangial expansion[109]. Both ob/ob and $\mathrm{db} / \mathrm{db}$ mice have been crossbreed with numerous genetically modified mice. Initially established by Zhao et al. the eNOS ${ }^{-/-} ; \mathrm{db} / \mathrm{db}$ mouse model has been identified as the most robust model of Type2 diabetes[110]. These mice display many human pathophysiological features of diabetic nephropathy including significant albuminuria (more than 30 -fold that of controls), reduction in glomerular filtration rate 
and glomerular basement membrane thickening[110].

The importance of performing animal studies in both male and female mice, as opposed to studies in one gender only (mostly male), is becoming increasingly apparent to improve the translation of novel therapies to both males and females. Recent findings showed that there were no significant differences in renal structural and functional damage between male and female diabetic eNOS $\mathrm{S}^{-/-} \mathrm{db} / \mathrm{db}$ mice [111]. Both male and female $\mathrm{eNOS}^{-/-} \mathrm{db} / \mathrm{db}$ mice showed hyperglycaemia and obesity as early as 8 weeks of age. Although male diabetic mice had a tendency towards greater body weights than female counterparts, the difference was not significant. Both male and female diabetic mice showed progressive albuminuria, renal hypertrophy, and significantly elevated serum creatinine, although this was slightly greater in female diabetic mice[111]. Notably, both male and female mice displayed significantly greater glomerular injury compared to their wildtype counterparts, but no differences were noted between genders. Hence, the eNOS ${ }^{-/-} \mathrm{db} / \mathrm{db}$ mouse model represents a robust model of diabetic nephropathy enabling the study of drug treatment in both male and female mice without gender impacting experimental outcomes. However, it should not be assumed that drug treatments will always necessitate the same outcomes, and for this reason both genders should be evaluated. However, historically male mice strains have been used to demonstrate more robust hyperglycaemia and renal injury.

Initially characterized by Clee et al., the black and tan, brachyuric (BTBR) mouse crossed onto the ob/ob background is a widely used mouse model to investigate Type2 diabetic nephropathy[112]. The BTBR mouse strain has alleles promoting insulin resistance resulting in natural hyperinsulinemia. When crossed with the ob/ob mouse, it develops a severe Type2 diabetic phenotype, maintaining sustained hyperglycaemia[112] and closely recapitulating the kidney lesions observed in human diabetic nephropathy. In particular, BTBR ob/ob mice exhibit glomerular hypertrophy, accumulation of mesangial matrix, loss of podocytes and glomerular lesions that are similar to that seen in human T2D kidney disease[113]. BTBR ob/ob mice display overactivation NF- $x \beta$ and JAK/STAT pathways, thereby promoting inflammation and the infiltration of lymphocytes and macrophages compared to BTBR wild type mice. Indeed, use of the BTBR ob/ob mouse model has demonstrated the therapeutic potential of targeting the transcription factor NF- $x ß$ in the prevention of diabetic nephropathy[114].

An additional, albeit invasive approach to develop diabetic nephropathy, is through the use of unilateral nephrectomy (UNx) that can significantly shorten the time required to develop the pathophysiological signs of diabetic nephropathy. This technique involves removing the left kidney after ligation of the renal artery, vein and ureter. Initially performed on STZ-induced diabetic Sprague-Dawley rats, it was later applied to mouse models to develop enhanced renal injury. Together with STZ, it has a synergistic effect by accelerating the development of glomerular lesions and impaired renal function. It has been shown that the combination of STZ and UNx is an effective way to induce diabetic nephropathy consistent with Type1 diabetes, especially in the resistant C57BL/6J mouse strain[115]. This model includes albuminuria, thickening of the glomerular basement membrane, mesangial matrix expansion, glomerular hypertrophy and upregulation of pro-fibrotic markers[115]. However, the use of a western-diet to induce Type2 diabetes was not effective in inducing significant albuminuria and glomerular injury when combined with STZ and UNx, despite the presence of hyperglycaemia. A possible explanation for this protective effect may have been driven by metabolic changes in the renal cells of the remaining kidney after UNx, where the hyperfunctioning nephron is able to avoid hypoxia-induced injury. Moreover, 15-weeks of a western diet may not have been sufficient to induce renal injury due to the high cholesterol, potentially providing protective effectives in the shorter-term $(<4$ months), therefore longer HFD-feeding may be required ( $~ 9$ months)[115].

More recently, the incorporation of proteomics and/or metabolomics studies is providing novel insights into the molecular pathways of diabetic nephropathy, as well as identifying mouse models that more closely phenocopy human diabetic kidney disease. Metabolomics analysis predominantly involves nuclear magnetic resonance spectroscopy or chromatography-mass spectrometry, enabling either the detection of known metabolites or numerous metabolites respectively, the latter technique without any prior knowledge of the metabolites required[116]. Using a proteomics and metabolomics approach, Rossi et al showed that a tissue 
inhibitor of metalloproteinases (TIMP3) knockout mouse model alone or in combination with STZ-mediated diabetes, led to significant alterations in kidney lipid metabolism and peripheral acylcartinine levels, which have been implicated in Type2 diabetes[117]. Loss of TIMP3 in the kidney was associated with features of diabetic nephropathy including higher levels of medium and long chain acylcarnitine in the blood. In contrast, levels of free carnitine and short chain beta-hydroxy and dicarboxy-acylcarnitines were lower diabetic in TIMP3 knockout mice as compared to WT-STZ mice[117]. Similar metabolomics signatures were found in a study by Lim et al who showed the accumulation of a number of short acylcarnitines in the plasma of patients who developed diabetic kidney disease over time, as well as data by Gurley et al who found elevated levels of short acylcarnitines in the Akita mouse[118], indicating that this model might closely resemble the human metabolic profile. Furthermore, the incorporation of flow cytometry allows the identification of immune cell-specific population in diabetic nephropathy. Indeed, a significant increase of intrarenal CD3+ $\mathrm{T}$ cells that was associated with augmented production of interferon- $\gamma$ and TNF- $\alpha$ in diabetic mice was revealed via flow cytometry[119]. In addition, transcriptomic profiling using scRNA-seq is identifying factors underlying the pathophysiology of diabetic nephropathy and is therefore a useful tool for identifying new therapeutic avenues for research. Using scRNA-seq, Fu et al demonstrated that the number of glomerular cell-specific markers that were significantly higher in diabetic mice compared with controls. These immune cells were predominantly macrophages and greater number expressed M1 phenotype than M2. Moreover, regulation of angiogenesis and migration pathways in endothelial cell from diabetic mice were altered[120]. Hence, these studies, in addition to adding valuable insights into the diseased kidney, also highlight the potential to generate a mouse model more prone to diabetic nephropathy.

\section{Diabetic retinopathy}

\subsubsection{Retinal pathophysiology: key features that need to be represented in animal models}

Diabetic retinopathy is the leading cause of visual impairment leading to blindness impacting approximately one third of the diabetic population[121]. Hyperglycaemia induced vascular damage involves several metabolic pathways analogous to those affecting diabetic nephropathy, including the polyol pathway, formation of AGEs and the PKC pathway. These pathways accelerate the loss of pericytes, apoptosis of endothelial cells, microaneurysms and thickening of the basement membrane, resulting in capillary occlusions and ischaemia. These processes trigger the upregulation of the growth factor VEGF, promoting vascular permeability and angiogenesis. This contributes to the progression of proliferative diabetic retinopathy and diabetic macular oedema, ultimately leading to vision loss and blindness[122].

\subsubsection{Models of diabetic retinopathy}

Rodent models are the most commonly used animal models to study diabetic retinopathy. Traditionally, the mouse model of retinopathy of prematurity (ROP) has been used as a surrogate model to study the formation of new malformed and leaky vessels, a hallmark of diabetic retinopathy. In this model, pups with their mothers are placed in an oxygen chamber $(75 \% \mathrm{O} 2 \pm 5 \%$ and $2 \% \mathrm{CO} 2$ for $22 \mathrm{~h} /$ day) at postnatal day 7 until postnatal day 11, after which they are returned to room air until day 18. The early exposure to elevated oxygen causes a central obliteration of the vasculature, whilst the return to room air mediates an hypoxia-driven increase in VEGF via HIF-1a resulting vascular neogenesis. Using this model, we have shown that oxidative stress is a key driver of neovascularisation[123], and that interventions like ebselen to bolster specific antioxidants such glutathione peroxidase-1[124] leads to significant attenuation of vasoobliteration and neovascularisation. In addition, by targeting the master regulator of oxidative stress, namely the transcription factor Nrf2 via the bardoxolone-methyl mimetic dh404, we additionally demonstrated that a global approach to bolstering antioxidant defence improved ROP in our mouse models[125].

To study diabetic retinopathy per se mouse or rat models can be used after exposure to STZ. Rat models (a single tail vein injection of STZ at $55 \mathrm{mg} / \mathrm{kg}$ with daily insulin) enable robust analysis of vascular leakage after 10-weeks of diabetes. In most experimental models, Sprague-Dawley rats are used for diabetic retinopathy studies. Gene and protein expression analysis of diabetic retinae enable the study of the molecular pathways involved, with emphasis on VEGF since this growth factor is the main causal factor in 
the vascular permeability that accompanies diabetic retinopathy. Similarly, the use of STZ has enabled the long-term study of diabetic retinopathy in mice. As mentioned earlier, female mice are known to be resistant to STZ. Recently, a study by Saadane et al have reported that the resistance of female C57BL/6J mice to STZ-induced diabetes can be overcome by injecting higher doses of STZ[26]. Female mice administered a dose of $75 \mathrm{mg} / \mathrm{kg}$ of body weight over 5 consecutive days, developed equivalent levels of hyperglycaemia as male counterparts administered $55 \mathrm{mg} / \mathrm{kg}$ of body weight over 5 consecutive days, which was maintained for at least 8 months, enabling a robust evaluation of diabetic retinopathy in this model. Importantly, both male and female diabetic mice developed similar abnormalities in retinal oxidative stress as measured by the level of superoxide in the retinae. This was accompanied by significantly increased expression of pro-inflammatory proteins, leucocyte mediated cytotoxicity and similarities in the number of degenerated capillaries in both male and female mice[26]. Thus, varying STZ dosage is an easy adjustment to enable the inclusion of both sexes in the exploration of therapeutic agents for complications such as diabetic retinopathy.

Inflammatory processes play a key role in driving diabetic retinopathy. To directly assess a role for inflammatory cytokines in the progression of diabetic retinopathy, Mugisho et al have developed a novel animal model that incorporates both hyperglycaemia and inflammation via single intravitreal injections of the pro-inflammatory cytokines, IL-1ß and TNF- $\alpha$, in non-obese diabetic (NOD) mice[126]. The injection of intravitreal cytokines evokes inflammation in the surrounding ocular tissue and exhibits retinal oedema, increased astrogliosis and microglia upregulation. In addition, pericyte loss observed by advanced vessel dilation and vessel beading, endothelial cell death and blood-retina barrier breakdown, are prominent[126]. The model may provide a new opportunity to further study the pathology of diabetic retinopathy, although its proneness to autoimmunity due to a genetic defect causing cytokine dysregulation could be a limitation. However, the effectiveness of this model was further highlighted in a follow-up study where connexcin43, an inhibitor of inflammation and inflammasome activation, was shown to significantly attenuate diabetic retinopathy[127].

\subsubsection{Emerging experimental analysis of diabetic retinopathy}

In most preclinical animal models, fluorescein angiography, optical coherence tomography and electroretinography are widely utilized methods to determine the functional changes of diabetic retinopathy. Additionally, inflammation and oxidative stress markers can be quantified by performing histology, real-time PCR, western blots, ELISA, immunohistochemistry and immunofluorescence using specific antibodies.

More recently, the discovery of a novel laser speckle flowgraphy imaging technique has emerged as a rapid and non-invasive method to determine basal blood flow and neuronal activity-dependent alterations in ocular diseases. Not only serving as a useful biomarker to detect and monitor postoperative outcomes for retinal diseases, this technique enables the detection of reduced blood flow volume and dysregulated retinal microvasculature that can cause hypoxia and eventually retinal neovascularization[128]. Moreover, the use of positron emission tomography (PET) imaging using $\left[{ }^{18} \mathrm{~F}\right] \mathrm{FP}-(+)-\mathrm{DTBZ}$ has been reported as an early diagnostic tool to evaluate diabetic retinopathy in rat models. The $\left[{ }^{18} \mathrm{~F}\right] \mathrm{FP}-(+)-\mathrm{DTBZ}$ PET targeting of vesicular monoamine transporter 2 (VMAT2) changes have been shown to detect dopaminergic neuron loss in the retina, a known characteristic symptom of early diabetic retinopathy[129].

A major limitation of current screening processes to detect diabetic retinopathy is that disease progression can go undetected until irreversible damage and blindness has occurred. The development of a biomarker for clinical screening would be a useful tool to overcome limitations such as the need for diagnosis by a qualified specialist, which can be costly, thereby restricting the population that can be effectively screened. To overcome this, Tawfik et al evaluated homocysteine (Hcy), a sulfur-containing amino acid known to induce the death of retinal ganglion cells, as a biomarker of diabetic retinopathy[130]. Indeed, an increase in Hcy levels was observed in the serum, vitreous humour and retina of both experimental animal models of diabetes and diabetic patients, while optical coherence tomography and fluorescein angiography has confirmed that Hcy-induced retinal changes in diabetic mice are aggravated[130]. In summary, Hcy should be investigated further as a strong biomarker candidate for diabetic retinopathy screening. 


\section{Diabetic neuropathy}

\subsubsection{Nerve pathophysiology: key features that need to be represented in animal models}

Diabetic neuropathy, characterised by damage to peripheral nerve function, is a highly prevalent complication of diabetes occurring in at least $6 \%-50 \%$ of diabetic patients depending on age and duration of diabetes[131, 132]. Diabetic patients often experience a high degree of morbidity, including poor balance and co-ordination leading to frequent falls, numbness, pain and ulceration of the lower limbs that can lead to limb amputation. Amongst the different forms of neuropathy, distal symmetric polyneuropathy commonly affecting the hands and lower limbs, is the most prevalent form of diabetic neuropathy. Hyperglycaemia induces accumulation of acylcarnitines that can trigger axonal degeneration. In addition, increased glucose levels lead to increased ROS, inflammation and AGE production, contributing to nervous system dysfunction[131]. Despite its high prevalence, the exact aetiology remains unknown, with available treatment options mostly ineffective, leaving the focus for patients mainly on improving glycaemic control or advocating for lifestyle modifications.

\subsubsection{Models of diabetic neuropathy}

Although animal models have been an invaluable tool in understanding the pathophysiology of diabetic vascular complications, the onset and progress of diabetic neuropathy in commonly used rodent models is not completely understood. Recent advances in animal models to study diabetic neuropathy have concentrated on models of Type2 diabetes. These advances have been made possible by creating models that are based on guidelines established by the Neurodiab Study Group of the European Association for the Study of Diabetes (NSGEASD) that determined that assessments of behaviour, motor and sensory nerve conduction velocity (NCVs), and nerve structure are essential requirements for a murine model of diabetic neuropathy[133].

Gregorio et al have characterized the onset and progression of Type2 diabetic neuropathy in the diabetic $\mathrm{db} / \mathrm{db}$ mouse on a C57BLKS/J background. These mice show hyperphagia[134] as well as other signs of diabetic neuropathy including progressive sensory loss, electrophysiological impairments, decreases in intraepidermal nerve fibre density, Schwann cells apoptosis and infiltration of T lymphocyte[135]. Other studies investigating neuropathy have been performed in $o b / o b$ leptin-based mouse models of type 2 diabetes[136].

A growing number of animal studies in recent years have demonstrated that low dose STZ combined with a high-fat diet can recapitulate certain features of Type 2 diabetes as seen in humans. In addition, fructose is now recognised as a major contributor to the T2D epidemic. Barrière et al have developed a high fat/high fructose (HF/HF)-STZ model of Type2 diabetes in the rat as useful model to study the long-term complications of Type 2 diabetes, including diabetic neuropathy, nephropathy and retinopathy[137]. Monitored over a period of 56 weeks, the HF/HF-STZ rats exhibited an early prediabetic phase of hyperinsulinemia with moderate dysglycaemia, developing into late stage hyperglycaemia, normalisation of insulinemia, marked dyslipidaemia, hepatic fibrosis and ultimately pancreatic $\beta$-cell failure. With respect to neuropathy, the HF/HF-STZ rats developed a gradual increase in mechanical hyperalgesia (an intense response to pain indicative of nerve damage) as measured by decreased mechanical nociceptive thresholds and displayed profound tactile allodynia, where HF/HF-STZ rats experience increased pain to relatively minor stimuli compared with controls. These phenotypic changes were accompanied by significant increases in impaired myelinated fibres of the sciatic nerves, increases in mitochondrial vulnerability and functional reorganization within the spinal dorsal horn circuitry[137].

In order to develop a more robust model of early onset neuropathy, O'Brien et al have characterised the development of neuropathy over time in a diet-induced obesity (DIO)-STZ mouse model of adult-onset Type2 diabetes and compared this with a model of DIO alone, the latter indicative of adolescent pre-diabetes. This has enabled a detailed analysis of neurogenic changes that align with the natural progression from obesity and impaired glucose tolerance to overt type 2 diabetes in adolescent and adult mice respectively[138]. To establish this model, one cohort was fed a high-fat diet starting at 5 weeks of age, whilst a second cohort additionally received low dose STZ at 12 weeks of age $(75 \mathrm{mg} / \mathrm{kg}$ body weight for the first injection and $50 \mathrm{mg} / \mathrm{kg}$ body weight for the second injection, 72 hours later) to cause mild impairment of insulin secretion and mild hyperglycaemia. Comparisons between the 2 cohorts at 16, 24 and 36 weeks displayed similar 
levels of decreased motor and sensory NCVs as early as 16 weeks followed by hypoalgesia and cutaneous nerve fibre loss at the later timepoints, despite the two groups having vastly different metabolic profiles[138]. Hence, this dual animal model approach may be more useful in understanding neurological pathophysiology of the young, enabling the development of novel therapies to target early neurological changes that are much needed with the growing prevalence of obesity and neurological changes of adolescent and young adults.

A less well-known, albeit invasive approach to develop diabetic neuropathy can be achieved by sciatic nerve transection. Focusing on the changes occurring in sensory nerve fibres with initial degeneration and regeneration processes resulting in pain, Pham et al developed a Type2 diabetic mouse model using sciatic nerve transection to study pathogenesis and treatment of early diabetic neuropathy[139]. Type2 diabetes was induced by injection of STZ and nicotinamide (NA) which was used to partially protect pancreatic $\beta$ cells from the toxic effects of STZ. This allowed the diabetic status to be more stable over a longer period of time, ensuring a high survival rate of the animals[140]. Sciatic nerve transection involves exposing the right sciatic nerve and inserting a silicone tube into the proximal and distal stumps leaving a gap between them. This model enables the evaluation of nerve regeneration in the same animal over several months. Of particular importance, these animals display mechanical hyperalgesia and a decrease in the number of intra-epidermal nerve fibres[140]. Since an effective treatment for diabetic neuropathy involves the prevention of progressive nerve degeneration whilst simultaneously promoting nerve regeneration, using the nerve transection-regeneration model enables the study of effective treatments for pre-diabetic and early stage diabetic neuropathy.

\section{Conclusions and Perspectives}

Animal models of diabetic complications have been developed over a number of years to best replicate the changes observed in humans. The need to more closely recapitulate critical aspects of the disease led to the establishment of the Animal Models of Diabetic Complications Consortium (AMDCC) in 2001 by the National Institutes of Health, with a guidelines paper published in 2007[141] specifically focused on the evaluation of cardiovascular complications. The goals of the AMDCC were to best characterize and validate various animal models of diabetic cardiovascular disease for basic, developmental, or translational research including outlining testing, prevention, early detection, therapy, and diagnostic imaging strategies. Since then, other consortia (e.g. NSGEASD: neuropathy; NIDDK Diab Complications Consortium (DiaComp): nephropathy, uropathy, retinopathy, wound healing, gastro-intestinal and liver disease, cardiovascular disease) have made available useful resources to standardise experimental protocols (https://www.diacomp.org). In this review, we have focussed on more recent advances as well as modifications to older methods that are allowing more nuanced analyses of diabetic micro and macrovascular complications. Careful choice of rodent model, as well as the mode of diabetes induction, duration of disease and window of study, whether that be younger (pre-diabetic models) or older more established T2D diabetic models, are all critical considerations that may impact the outcomes of a study. For example, this review has emphasized the appropriateness of using STZ in the induction of T1D with caveats around dose, gender, off-target toxicity and alterations in gut microbiota. In addition, this review has highlighted relevant murine models for both micro- and macrovascular complications and the need for the inclusion of both male and female models. Additionally, where appropriate, limitations of the models have been noted. These considerations will assist in effective study design to maximise results for the evaluation of novel therapies to limit these debilitating complications.

\section{References}

1. Low Wang, C.C., et al., Clinical Update: Cardiovascular Disease in Diabetes Mellitus: Atherosclerotic Cardiovascular Disease and Heart Failure in Type 2 Diabetes Mellitus - Mechanisms, Management, and Clinical Considerations. Circulation, 2016. 133 (24): p. 2459-502.

2. Kosiborod, M., et al., Vascular complications in patients with type 2 diabetes: prevalence and associated factors in 38 countries (the DISCOVER study program). Cardiovasc Diabetol, 2018. 17 (1): p. 150.

3. Leon, B.M. and T.M. Maddox, Diabetes and cardiovascular disease: Epidemiology, biological mechanisms, treatment recommendations and future research. World J Diabetes, 2015. 6 (13): p. 1246-58. 
4. Chokshi, N.P., E. Grossman, and F.H. Messerli, Blood pressure and diabetes: vicious twins. Heart, 2013. 99 (8): p. $577-85$.

5. de Boer, I.H., et al., Diabetes and Hypertension: A Position Statement by the American Diabetes Association. Diabetes Care, 2017.40 (9): p. 1273-1284.

6. Geovanini, G.R. and P. Libby, Atherosclerosis and inflammation: overview and updates. Clin Sci (Lond), 2018. 132 (12): p. 1243-1252.

7. Katakami, N., Mechanism of Development of Atherosclerosis and Cardiovascular Disease in Diabetes Mellitus. J Atheroscler Thromb, 2018. 25 (1): p. 27-39.

8. Sourris, K.C., B.E. Harcourt, and J.M. Forbes, A new perspective on therapeutic inhibition of advanced glycation in diabetic microvascular complications: common downstream endpoints achieved through disparate therapeutic approaches? Am J Nephrol, 2009.30 (4): p. 323-35.

9. Schleicher, E. and U. Friess, Oxidative stress, AGE, and atherosclerosis. Kidney Int Suppl, 2007(106): p. S17-26.

10. Goldberg, I.J., Clinical review 124: Diabetic dyslipidemia: causes and consequences. J Clin Endocrinol Metab, 2001. 86 (3): p. 965-71.

11. Goldberg, I.J., et al., Decreased lipoprotein clearance is responsible for increased cholesterol in LDL receptor knockout mice with streptozotocin-induced diabetes. Diabetes, 2008. 57 (6): p. 1674-82.

12. Oppi, S., T.F. Luscher, and S. Stein, Mouse Models for Atherosclerosis Research-Which Is My Line? Front Cardiovasc Med, 2019.6 : p. 46.

13. Tanigawa, H., et al., Expression of cholesteryl ester transfer protein in mice promotes macrophage reverse cholesterol transport.Circulation, 2007. 116 (11): p. 1267-73.

14. VanderLaan, P.A., C.A. Reardon, and G.S. Getz, Site specificity of atherosclerosis: site-selective responses to atherosclerotic modulators. Arterioscler Thromb Vasc Biol, 2004.24 (1): p. 12-22.

15. Park, L., et al., Suppression of accelerated diabetic atherosclerosis by the soluble receptor for advanced glycation endproducts. Nat Med, 1998. 4 (9): p. 1025-31.

16. Szkudelski, T., The mechanism of alloxan and streptozotocin action in B cells of the rat pancreas. Physiol Res, 2001.50 (6): p. 537-46.

17. Gray, S.P., et al., Reactive Oxygen Species Can Provide Atheroprotection via NOX4-Dependent Inhibition of Inflammation and Vascular Remodeling. Arterioscler Thromb Vasc Biol, 2016.36 (2): p. 295-307.

18. Brennan, E., et al., Protective Effect of let-7 miRNA Family in Regulating Inflammation in DiabetesAssociated Atherosclerosis.Diabetes, 2017. 66 (8): p. 2266-2277.

19. Tikellis, C., et al., Dicarbonyl stress in the absence of hyperglycemia increases endothelial inflammation and atherogenesis similar to that observed in diabetes. Diabetes, 2014. 63 (11): p. 3915-25.

20. Sharma, A., et al., Direct Endothelial Nitric Oxide Synthase Activation Provides Atheroprotection in Diabetes-Accelerated Atherosclerosis. Diabetes, 2015. 64 (11): p. 3937-50.

21. Tan, S.M., et al., The modified selenenyl amide, M-hydroxy ebselen, attenuates diabetic nephropathy and diabetes-associated atherosclerosis in ApoE/GPx1 double knockout mice. PLoS One, 2013.8 (7): p. e69193.

22. Tan, S.M., et al., Derivative of bardoxolone methyl, dh404, in an inverse dose-dependent manner lessens diabetes-associated atherosclerosis and improves diabetic kidney disease. Diabetes, 2014.63 (9): p. 3091-103.

23. Chow, F.Y., et al., Monocyte chemoattractant protein-1 promotes the development of diabetic renal injury in streptozotocin-treated mice. Kidney Int, 2006. 69 (1): p. 73-80. 
24. de Haan, J.B., et al., Kidney expression of glutathione peroxidase-1 is not protective against streptozotocin-induced diabetic nephropathy. Am J Physiol Renal Physiol, 2005. 289 (3): p. F544-51.

25. Gvazava, I.G., et al., Pathogenesis of Type 1 Diabetes Mellitus and Rodent Experimental Models. Acta Naturae, 2018.10 (1): p. 24-33.

26. Saadane, A., et al., Successful induction of diabetes in mice demonstrates no gender difference in development of early diabetic retinopathy. PLoS One, 2020. 15 (9): p. e0238727.

27. Costa, F.R., et al., Gut microbiota translocation to the pancreatic lymph nodes triggers NOD2 activation and contributes to T1D onset. J Exp Med, 2016. 213 (7): p. 1223-39.

28. Soro-Paavonen, A., et al., Receptor for advanced glycation end products (RAGE) deficiency attenuates the development of atherosclerosis in diabetes. Diabetes, 2008. 57 (9): p. 2461-9.

29. Calkin, A.C., et al., Gemfibrozil decreases atherosclerosis in experimental diabetes in association with a reduction in oxidative stress and inflammation. Diabetologia, 2006. 49 (4): p. 766-74.

30. Graham, T.L., et al., The PPARdelta agonist GW0742X reduces atherosclerosis in LDLR(-/-) mice. Atherosclerosis, 2005.181 (1): p. 29-37.

31. Al-Sharea, A., et al., SGLT2 inhibition reduces atherosclerosis by enhancing lipoprotein clearance in Ldlr(-/-) type 1 diabetic mice. Atherosclerosis, 2018. 271 : p. 166-176.

32. Morgantini, C., et al., Apolipoprotein A-I mimetic peptides prevent atherosclerosis development and reduce plaque inflammation in a murine model of diabetes. Diabetes, 2010. 59 (12): p. 3223-8.

33. Keren, P., et al., Effect of hyperglycemia and hyperlipidemia on atherosclerosis in LDL receptor-deficient mice: establishment of a combined model and association with heat shock protein 65 immunity.Diabetes, 2000. 49 (6): p. 1064-9.

34. Toque, H.A., et al., Akita spontaneously type 1 diabetic mice exhibit elevated vascular arginase and impaired vascular endothelial and nitrergic function. PLoS One, 2013. 8 (8): p. e72277.

35. Jun, J.Y., Z. Ma, and L. Segar, Spontaneously diabetic Ins2(+/Akita):apoE-deficient mice exhibit exaggerated hypercholesterolemia and atherosclerosis. Am J Physiol Endocrinol Metab, 2011. 301 (1): p. E145-54.

36. Engelbertsen, D., et al., Increased inflammation in atherosclerotic lesions of diabetic Akita-LDLr(-)/(-) mice compared to nondiabetic LDLr(-)/(-) mice. Exp Diabetes Res, 2012. 2012 : p. 176162.

37. Zhou, C., et al., Hyperglycemic Ins2AkitaLdlr(-)/(-) mice show severely elevated lipid levels and increased atherosclerosis: a model of type 1 diabetic macrovascular disease. J Lipid Res, 2011.52 (8): p. 1483-93.

38. Vikramadithyan, R.K., et al., Human aldose reductase expression accelerates diabetic atherosclerosis in transgenic mice. J Clin Invest, 2005. 115 (9): p. 2434-43.

39. Johansson, F., et al., Type 1 diabetes promotes disruption of advanced atherosclerotic lesions in LDL receptor-deficient mice. Proc Natl Acad Sci U S A, 2008. 105 (6): p. 2082-7.

40. Renard, C.B., et al., Diabetes and diabetes-associated lipid abnormalities have distinct effects on initiation and progression of atherosclerotic lesions. J Clin Invest, 2004. 114 (5): p. 659-68.

41. Tabish, S.A., Is Diabetes Becoming the Biggest Epidemic of the Twenty-first Century? Int J Health Sci (Qassim), 2007. 1 (2): p. V-VIII.

42. Wu, K.K., et al., Increased hypercholesterolemia and atherosclerosis in mice lacking both ApoE and leptin receptor.Atherosclerosis, 2005. 181 (2): p. 251-9.

43. Wendt, T., et al., RAGE modulates vascular inflammation and atherosclerosis in a murine model of type 2 diabetes. Atherosclerosis, 2006. 185 (1): p. 70-7. 
44. Lloyd, D.J., et al., Generation and characterization of two novel mouse models exhibiting the phenotypes of the metabolic syndrome: Apob48-/-Lepob/ob mice devoid of ApoE or Ldlr. Am J Physiol Endocrinol Metab, 2008. 294 (3): p. E496-505.

45. Veniant, M.M., et al., Lipoprotein clearance mechanisms in LDL receptor-deficient "Apo-B48-only" and "Apo-B100-only" mice. J Clin Invest, 1998. 102 (8): p. 1559-68.

46. Heinonen, S.E., et al., Increased atherosclerotic lesion calcification in a novel mouse model combining insulin resistance, hyperglycemia, and hypercholesterolemia. Circ Res, 2007.101 (10): p. 1058-67.

47. Burke, A.P., et al., Morphologic findings of coronary atherosclerotic plaques in diabetics: a postmortem study. Arterioscler Thromb Vasc Biol, 2004. 24 (7): p. 1266-71.

48. Godsland, I.F., et al., Coronary calcification, homocysteine, C-reactive protein and the metabolic syndrome in Type 2 diabetes: the Prospective Evaluation of Diabetic Ischaemic Heart Disease by Coronary Tomography (PREDICT) Study. Diabet Med, 2006. 23 (11): p. 1192-200.

49. Wagenknecht, L.E., et al., Familial aggregation of coronary artery calcium in families with type 2 diabetes. Diabetes, 2001.50 (4): p. 861-6.

50. Gonzalez-Navarro, H., et al., Plasma insulin levels predict the development of atherosclerosis when IRS2 deficiency is combined with severe hypercholesterolemia in apolipoprotein E-null mice. Front Biosci, 2007. 12 : p. 2291-8.

51. Baumgartl, J., et al., Myeloid lineage cell-restricted insulin resistance protects apolipoproteinE-deficient mice against atherosclerosis. Cell Metab, 2006. 3 (4): p. 247-56.

52. Galkina, E.V., et al., Accelerated atherosclerosis in Apoe-/- mice heterozygous for the insulin receptor and the insulin receptor substrate-1. Arterioscler Thromb Vasc Biol, 2012. 32 (2): p. 247-56.

53. Goodyear, L.J., et al., Insulin receptor phosphorylation, insulin receptor substrate-1 phosphorylation, and phosphatidylinositol 3-kinase activity are decreased in intact skeletal muscle strips from obese subjects. J Clin Invest, 1995. 95 (5): p. 2195-204.

54. Gonzalez-Navarro, H., et al., Molecular mechanisms of atherosclerosis in metabolic syndrome: role of reduced IRS2-dependent signaling. Arterioscler Thromb Vasc Biol, 2008. 28 (12): p. 2187-94.

55. Adingupu, D.D., et al., Hyperglycemia Induced by Glucokinase Deficiency Accelerates Atherosclerosis Development and Impairs Lesion Regression in Combined Heterozygous Glucokinase and the Apolipoprotein E-Knockout Mice. J Diabetes Res, 2016. 2016 : p. 8630961.

56. Schreyer, S.A., et al., LDL receptor but not apolipoprotein E deficiency increases diet-induced obesity and diabetes in mice. Am J Physiol Endocrinol Metab, 2002. 282 (1): p. E207-14.

57. Schreyer, S.A., et al., Mice deficient in apolipoprotein $E$ but not LDL receptors are resistant to accelerated atherosclerosis associated with obesity. Atherosclerosis, 2003. 171 (1): p. 49-55.

58. Phillips, J.W., et al., Rosiglitazone reduces the accelerated neointima formation after arterial injury in a mouse injury model of type 2 diabetes. Circulation, 2003. 108 (16): p. 1994-9.

59. Subramanian, S., et al., Dietary cholesterol worsens adipose tissue macrophage accumulation and atherosclerosis in obese LDL receptor-deficient mice. Arterioscler Thromb Vasc Biol, 2008.28 (4): p. 685-91.

60. Kanter, J.E., et al., A Novel Strategy to Prevent Advanced Atherosclerosis and Lower Blood Glucose in a Mouse Model of Metabolic Syndrome. Diabetes, 2018. 67 (5): p. 946-959.

61. Neuhofer, A., et al., An accelerated mouse model for atherosclerosis and adipose tissue inflammation. Cardiovasc Diabetol, 2014. 13 : p. 23. 
62. Cannizzo, B., et al., Insulin resistance promotes early atherosclerosis via increased proinflammatory proteins and oxidative stress in fructose-fed ApoE-KO mice. Exp Diabetes Res, 2012.2012 : p. 941304.

63. Sanguesa, G., et al., The Addition of Liquid Fructose to a Western-Type Diet in LDL-R(-/-) Mice Induces Liver Inflammation and Fibrogenesis Markers without Disrupting Insulin Receptor Signalling after an Insulin Challenge. Nutrients, 2017. 9 (3).

64. Suman, R.K., et al., Development of an Experimental Model of Diabetes Co-Existing with Metabolic Syndrome in Rats. Adv Pharmacol Sci, 2016. 2016 : p. 9463476.

65. Tate, M., et al., Characterising an Alternative Murine Model of Diabetic Cardiomyopathy. Front Physiol, 2019. 10 : p. 1395.

66. Gargiulo, S., M. Gramanzini, and M. Mancini, Molecular Imaging of Vulnerable Atherosclerotic Plaques in Animal Models. Int J Mol Sci, 2016. 17 (9).

67. Schwartz, S.M. and K.E. Bornfeldt, How does diabetes accelerate atherosclerotic plaque rupture and arterial occlusion? Front Biosci, 2003. 8 : p. s1371-83.

68. Lima, L.C., et al., Mononuclear cell therapy reverts cuff-induced thrombosis in apolipoprotein E-deficient mice. Lipids Health Dis, 2012. 11 : p. 96.

69. Mazzolai, L., et al., Endogenous angiotensin II induces atherosclerotic plaque vulnerability and elicits a Th1 response in ApoE-/- mice. Hypertension, 2004. 44 (3): p. 277-82.

70. Daugherty, A., M.W. Manning, and L.A. Cassis, Angiotensin II promotes atherosclerotic lesions and aneurysms in apolipoprotein E-deficient mice. J Clin Invest, 2000. 105 (11): p. 1605-12.

71. Van Herck, J.L., et al., Impaired fibrillin-1 function promotes features of plaque instability in apolipoprotein E-deficient mice. Circulation, 2009. 120 (24): p. 2478-87.

72. Van der Donckt, C., et al., Elastin fragmentation in atherosclerotic mice leads to intraplaque neovascularization, plaque rupture, myocardial infarction, stroke, and sudden death. Eur Heart J, 2015. 36 (17): p. 1049-58.

73. Chen, Y.C., J. Rivera, and K. Peter, Tandem Stenosis to Induce Atherosclerotic Plaque Instability in the Mouse. Methods Mol Biol, 2015. 1339 : p. 333-8.

74. Paigen, B., et al., Quantitative assessment of atherosclerotic lesions in mice. Atherosclerosis, 1987. 68 (3): p. 231-40.

75. Daugherty, A. and S.C. Whitman, Quantification of atherosclerosis in mice. Methods Mol Biol, 2003. 209 : p. 293-309.

76. Gray, S.P., et al., NADPH oxidase 1 plays a key role in diabetes mellitus-accelerated atherosclerosis. Circulation, 2013.127 (18): p. 1888-902.

77. Chew, P., et al., Site-specific antiatherogenic effect of the antioxidant ebselen in the diabetic apolipoprotein E-deficient mouse.Arterioscler Thromb Vasc Biol, 2009. 29 (6): p. 823-30.

78. Chew, P., et al., Antiatherosclerotic and renoprotective effects of ebselen in the diabetic apolipoprotein E/GPx1-double knockout mouse. Diabetes, 2010. 59 (12): p. 3198-207.

79. Al-Sharea, A., et al., Chronic sympathetic driven hypertension promotes atherosclerosis by enhancing hematopoiesis. Haematologica, 2019. 104 (3): p. 456-467.

80. Sharma, A., P.N. Bernatchez, and J.B. de Haan, Targeting endothelial dysfunction in vascular complications associated with diabetes. Int J Vasc Med, 2012. 2012 : p. 750126.

81. Rasmiena, A.A., et al., Plasmalogen modulation attenuates atherosclerosis in ApoE- and ApoE/GPx1deficient mice. Atherosclerosis, 2015. 243 (2): p. 598-608. 
82. Martinez, H.G., et al., Images in cardiovascular medicine: Microscopic computed tomography-based virtual histology for visualization and morphometry of atherosclerosis in diabetic apolipoprotein e mutant mice. Circulation, 2009. 120 (9): p. 821-2.

83. McAteer, M.A., et al., Quantification and 3D reconstruction of atherosclerotic plaque components in apolipoprotein E knockout mice using ex vivo high-resolution MRI. Arterioscler Thromb Vasc Biol, 2004.24 (12): p. 2384-90.

84. Htun, N.M., et al., Near-infrared autofluorescence induced by intraplaque hemorrhage and heme degradation as marker for high-risk atherosclerotic plaques. Nat Commun, 2017. 8 (1): p. 75.

85. Espina, V., et al., Laser-capture microdissection. Nat Protoc, 2006. 1 (2): p. 586-603.

86. Whitman, S.C., A practical approach to using mice in atherosclerosis research. Clin Biochem Rev, 2004. 25 (1): p. 81-93.

87. Feig, J.E. and E.A. Fisher, Laser capture microdissection for analysis of macrophage gene expression from atherosclerotic lesions.Methods Mol Biol, 2013. 1027 : p. 123-35.

88. Gjurich, B.N., P.L. Taghavie-Moghadam, and E.V. Galkina, Flow Cytometric Analysis of Immune Cells Within Murine Aorta. Methods Mol Biol, 2015. 1339 : p. 161-75.

89. Bonanno, E., et al., Flow cytometry analysis of atherosclerotic plaque cells from human carotids: a validation study.Cytometry, 2000. 39 (2): p. 158-65.

90. Nagareddy, P.R., et al., Hyperglycemia promotes myelopoiesis and impairs the resolution of atherosclerosis. Cell Metab, 2013.17 (5): p. 695-708.

91. Brennan, E.P., et al., Lipoxins Protect Against Inflammation in Diabetes-Associated Atherosclerosis. Diabetes, 2018.67 (12): p. 2657-2667.

92. Lebedeva, A., et al., Ex vivo culture of human atherosclerotic plaques: A model to study immune cells in atherogenesis.Atherosclerosis, 2017. 267 : p. 90-98.

93. Tang, X., et al., The single-cell sequencing: new developments and medical applications. Cell Biosci, 2019. 9 : p. 53.

94. Depuydt, M.A.C., et al., Microanatomy of the Human Atherosclerotic Plaque by Single-Cell Transcriptomics. Circ Res, 2020.127 (11): p. 1437-1455.

95. Cochain, C., et al., Single-Cell RNA-Seq Reveals the Transcriptional Landscape and Heterogeneity of Aortic Macrophages in Murine Atherosclerosis. Circ Res, 2018.122 (12): p. 1661-1674.

96. Zhang, H., et al., First Giant Steps Toward a Cell Atlas of Atherosclerosis. Circ Res, 2018.122 (12): p. $1632-1634$.

97. Fernandez, D.M., et al., Single-cell immune landscape of human atherosclerotic plaques. Nat Med, 2019. 25 (10): p. 1576-1588.

98. Michell, D.L., et al., Imaging leukocyte adhesion to the vascular endothelium at high intraluminal pressure. J Vis Exp, 2011(54).

99. Pickering, R.J., et al., Transactivation of RAGE mediates angiotensin-induced inflammation and atherogenesis. J Clin Invest, 2019. 129 (1): p. 406-421.

100. Schofield, I., et al., Vascular structural and functional changes in type 2 diabetes mellitus: evidence for the roles of abnormal myogenic responsiveness and dyslipidemia. Circulation, 2002.106 (24): p. $3037-43$.

101. Breyer, M.D., et al., Mouse models of diabetic nephropathy.J Am Soc Nephrol, 2005.16 (1): p. 27-45. 
102. Robinson, R., et al., Update on animal models of diabetic retinopathy: from molecular approaches to mice and higher mammals. Dis Model Mech, 2012. 5 (4): p. 444-56.

103. O'Brien, P.D., S.A. Sakowski, and E.L. Feldman, Mouse models of diabetic neuropathy. ILAR J, 2014. 54 (3): p. 259-72.

104. Fowler, M.J., Microvascular and Macrovascular Complications of Diabetes. Clinical Diabetes, 2008. 26 (2): p. $77-82$.

105. Cade, W.T., Diabetes-related microvascular and macrovascular diseases in the physical therapy setting. Phys Ther, 2008.88 (11): p. 1322-35.

106. Alicic, R.Z., M.T. Rooney, and K.R. Tuttle, Diabetic Kidney Disease: Challenges, Progress, and Possibilities. Clin J Am Soc Nephrol, 2017. 12 (12): p. 2032-2045.

107. Park, K.M., et al., A novel mouse model of diabetes mellitus using unilateral nephrectomy. Lab Anim, 2016. 50 (2): p. 88-93.

108. Huang, C., et al., The KCa3.1 blocker TRAM34 reverses renal damage in a mouse model of established diabetic nephropathy. PLoS One, 2018. 13 (2): p. e0192800.

109. Sharma, K., P. McCue, and S.R. Dunn, Diabetic kidney disease in the $d b / d b$ mouse. Am J Physiol Renal Physiol, 2003. 284 (6): p. F1138-44.

110. Zhao, H.J., et al., Endothelial nitric oxide synthase deficiency produces accelerated nephropathy in diabetic mice. J Am Soc Nephrol, 2006. 17 (10): p. 2664-9.

111. Ma, Y., et al., Comparison of diabetic nephropathy between male and female eNOS(-/-) db/db mice. Am J Physiol Renal Physiol, 2019.316 (5): p. F889-F897.

112. Clee, S.M., S.T. Nadler, and A.D. Attie, Genetic and genomic studies of the BTBR ob/ob mouse model of type 2 diabetes. Am J Ther, 2005. 12 (6): p. 491-8.

113. Hudkins, K.L., et al., BTBR Ob/Ob mutant mice model progressive diabetic nephropathy. J Am Soc Nephrol, 2010.21 (9): p. 1533-42.

114. Opazo-Rios, L., et al., Targeting NF-kappaB by the Cell-Permeable NEMO-Binding Domain Peptide Improves Albuminuria and Renal Lesions in an Experimental Model of Type 2 Diabetic Nephropathy.Int J Mol Sci, 2020. 21 (12).

115. Uil, M., et al., Combining streptozotocin and unilateral nephrectomy is an effective method for inducing experimental diabetic nephropathy in the 'resistant' C57Bl/6J mouse strain. Sci Rep, 2018.8 (1): p. 5542.

116. Abbiss, H., G.L. Maker, and R.D. Trengove, Metabolomics Approaches for the Diagnosis and Understanding of Kidney Diseases.Metabolites, 2019. 9 (2).

117. Rossi, C., et al., Proteomic and metabolomic characterization of streptozotocin-induced diabetic nephropathy in TIMP3-deficient mice.Acta Diabetol, 2018. 55 (2): p. 121-129.

118. Azushima, K., S.B. Gurley, and T.M. Coffman, Modelling diabetic nephropathy in mice. Nat Rev Nephrol, 2018. 14 (1): p. 48-56.

119. Moon, J.Y., et al., Aberrant recruitment and activation of $T$ cells in diabetic nephropathy. Am J Nephrol, 2012. 35 (2): p. 164-74.

120. Fu, J., et al., Single-Cell RNA Profiling of Glomerular Cells Shows Dynamic Changes in Experimental Diabetic Kidney Disease. J Am Soc Nephrol, 2019. 30 (4): p. 533-545.

121. Yau, J.W., et al., Global prevalence and major risk factors of diabetic retinopathy. Diabetes Care, 2012. 35 (3): p. 556-64. 
122. Duh, E.J., J.K. Sun, and A.W. Stitt, Diabetic retinopathy: current understanding, mechanisms, and treatment strategies. JCI Insight, 2017. 2 (14).

123. Tan, S.M., et al., Lack of the antioxidant glutathione peroxidase-1 (GPx1) exacerbates retinopathy of prematurity in mice.Invest Ophthalmol Vis Sci, 2013. 54 (1): p. 555-62.

124. Tan, S.M., et al., Ebselen by modulating oxidative stress improves hypoxia-induced macroglial Muller cell and vascular injury in the retina. Exp Eye Res, 2015. 136 : p. 1-8.

125. Deliyanti, D., et al., A potent Nrf2 activator, dh404, bolsters antioxidant capacity in glial cells and attenuates ischaemic retinopathy. Clin Sci (Lond), 2016. 130 (15): p. 1375-87.

126. Mugisho, O.O., et al., Intravitreal pro-inflammatory cytokines in non-obese diabetic mice: Modelling signs of diabetic retinopathy. PLoS One, 2018. 13 (8): p. e0202156.

127. Mugisho, O.O., et al., Connexin43 hemichannel block protects against the development of diabetic retinopathy signs in a mouse model of the disease. J Mol Med (Berl), 2019. 97 (2): p. 215-229.

128. Chaurasia, S.S., et al., The NLRP3 Inflammasome May Contribute to Pathologic Neovascularization in the Advanced Stages of Diabetic Retinopathy. Sci Rep, 2018. 8 (1): p. 2847.

129. Li, J., et al., PET Imaging of Vesicular Monoamine Transporter 2 in Early Diabetic Retinopathy Using [(18)F]FP-(+)-DTBZ. Mol Imaging Biol, 2020. 22 (5): p. 1161-1169.

130. Tawfik, A., et al., Homocysteine: A Potential Biomarker for Diabetic Retinopathy. J Clin Med, 2019. 8 (1).

131. Feldman, E.L., et al., Diabetic neuropathy. Nat Rev Dis Primers, 2019. 5 (1): p. 41.

132. Hicks, C.W. and E. Selvin, Epidemiology of Peripheral Neuropathy and Lower Extremity Disease in Diabetes. Curr Diab Rep, 2019. 19 (10): p. 86.

133. Biessels, G.J., et al., Phenotyping animal models of diabetic neuropathy: a consensus statement of the diabetic neuropathy study group of the EASD (Neurodiab). J Peripher Nerv Syst, 2014. 19 (2): p. 77-87.

134. Sullivan, K.A., et al., Mouse models of diabetic neuropathy.Neurobiol Dis, 2007. 28 (3): p. 276-85.

135. De Gregorio, C., et al., Characterization of diabetic neuropathy progression in a mouse model of type 2 diabetes mellitus.Biol Open, 2018. 7 (9).

136. O'Brien, P.D., et al., BTBR ob/ob mice as a novel diabetic neuropathy model: Neurological characterization and gene expression analyses. Neurobiol Dis, 2015. 73 : p. 348-55.

137. Barriere, D.A., et al., Combination of high-fat/high-fructose diet and low-dose streptozotocin to model long-term type-2 diabetes complications. Sci Rep, 2018. 8 (1): p. 424.

138. O'Brien, P.D., et al., Juvenile murine models of prediabetes and type 2 diabetes develop neuropathy. Dis Model Mech, 2018.11 (12).

139. Pham, V.M., et al., Impaired peripheral nerve regeneration in type-2 diabetic mouse model. Eur J Neurosci, 2018. 47 (2): p. 126-139.

140. Szkudelski, T., Streptozotocin-nicotinamide-induced diabetes in the rat. Characteristics of the experimental model. Exp Biol Med (Maywood), 2012. 237 (5): p. 481-90.

141. Hsueh, W., et al., Recipes for creating animal models of diabetic cardiovascular disease. Circ Res, 2007. 100 (10): p. 1415-27.

Figures and Tables 


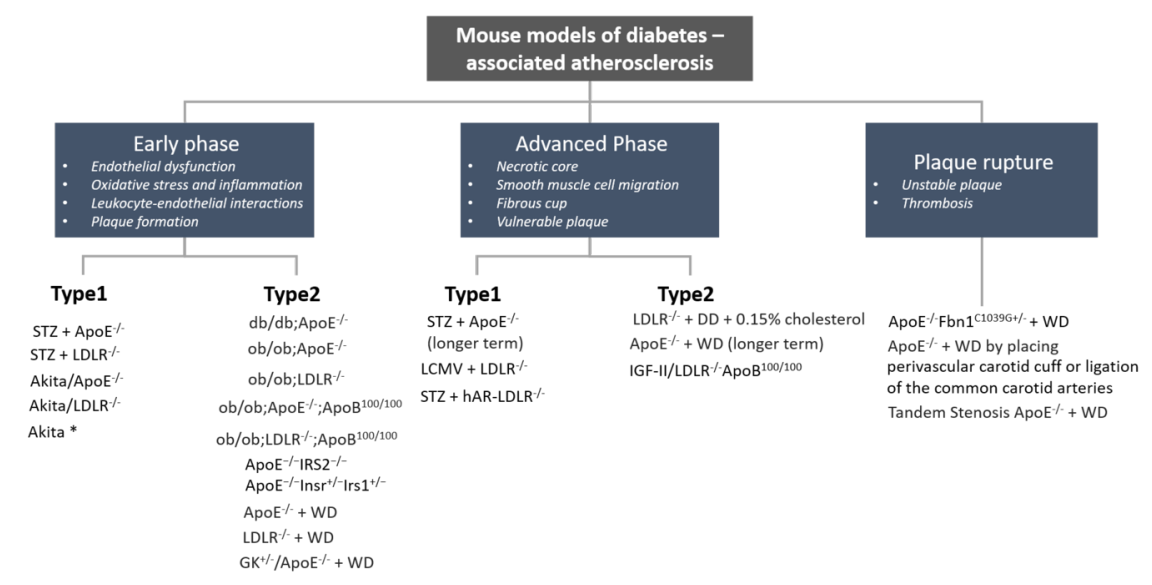

Figure 1: Summary of common mouse models of diabetes-associated atherosclerosis at various stages of the disease. STZ: streptozotocin; ApoE: Apolipoprotein E; LDLR: Low density lipoprotein receptor; $\mathrm{db} / \mathrm{db}$ : leptin receptor deficient; ob/ob: leptin deficient; ApoB ${ }^{100 / 100}$ : Apoliprotein B100; IRS: Insulin receptor substrate; Insr: Insulin receptor; WD: Western Diet; GK: Glucokinase; LCMV: Lymphocytic choriomeningitis virus; hAR: Human aldose reductase; DD: diabetogenic diet; IGF-11: Insulin growth factor II; Fbn ${ }^{\mathrm{C1093G}+/-}$ : Fibrillin-1 habouring a heterozygous C1093G. * Model of endothelial dysfunction.

\begin{tabular}{|c|c|c|c|}
\hline Analysis & Technique & Research Output & Reference \\
\hline Plaque visualisation & $\begin{array}{l}\text { MicroCT } \\
\text { - } 3 \text { M MRI } \\
\text { comporescence emission } \\
\text { Infra-red range) }\end{array}$ & $\begin{array}{l}\text { Volumetric 3D assessment } \\
\text { Degree of occlusion and } \\
\text { instability }\end{array}$ & {$[82],[83],[84]$} \\
\hline Plaque quantification & $\begin{array}{ll}\cdot & \text { En face Sudan IV } \\
\text { - } & \text { Oil red O (aortic sinus) }\end{array}$ & Lesion area & [74] \\
\hline Plaque cellular composition & $\begin{array}{ll}\text { - } & \text { H\&E } \\
& \text { Picosirius Red } \\
\text { - } & \text { Masson's Trichrome } \\
\text { - } \mathrm{IHC} / \mathrm{IF}\end{array}$ & $\begin{array}{l}\text { - } \begin{array}{l}\text { Necrotic core } \\
\text { - }\end{array} \text { Follagen content } \\
\text { morous plaque and } \\
\text { SMC, endothelial cells, } \\
\text { macrophages }\end{array}$ & [31], [76-79] \\
\hline $\begin{array}{l}\text { Inflammatory and oxidative } \\
\text { stress markers }\end{array}$ & $\begin{array}{ll}\text { - } & \text { Western Blot } \\
\text { - } & \text { IHC/IF } \\
\text { - } & \text { ELISA } \\
\text { : } & \text { PCR } \\
\text { - } & \text { LCM }\end{array}$ & $\begin{array}{l}\text { Specific antibodies can be } \\
\text { utilised to investigate protein } \\
\text { and gene expression of } \\
\text { inflammatory and oxidative } \\
\text { markers }\end{array}$ & {$[20],[76],[80],[81]$} \\
\hline $\begin{array}{l}\text { Cell-specific markers or } \\
\text { transcriptomes }\end{array}$ & $\begin{array}{l}\text { - Flow cytometry } \\
\text { - scRNAseq }\end{array}$ & $\begin{array}{l}\text { Immune cell specific } \\
\text { populations } \\
\text { - Transcriptomes/genetic } \\
\text { signatures }\end{array}$ & {$[88-90],[93-97]$} \\
\hline Endothelial dysfunction & - Vessel myography & $\begin{array}{ll}\text { - Endothelium-dependent } \\
\text { function } \\
\text { - Endothelium-independent } \\
\text { function } \\
\text { - Phenylephrine contractility } \\
\text { - Nitric oxide bioavailability }\end{array}$ & {$[20],[100]$} \\
\hline $\begin{array}{l}\text { Leukocyte-endothelial } \\
\text { interactions }\end{array}$ & - Intravital Microscopy & $\begin{array}{l}\text { Real time leukocyte rolling, } \\
\text { adhesion and quantification }\end{array}$ & [20], [98], [99] \\
\hline
\end{tabular}

Table 1: Summary of experimental analysis and research output for models of diabetes-associated atherosclerosis. 


\begin{tabular}{|c|c|c|c|c|}
\hline & Analysis & Technique & Research Output & Reference \\
\hline \multirow{5}{*}{ 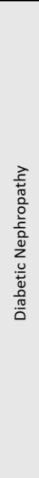 } & Molecular pathways & $\begin{array}{ll}\text { - } & \text { Proteomics } \\
\text { Metabolomics }\end{array}$ & $\begin{array}{l}\text { - Alteration in metabolic } \\
\text { pathways }\end{array}$ & {$[116],[117]$} \\
\hline & $\begin{array}{l}\text { Cell-specific markers or } \\
\text { transcriptomes }\end{array}$ & $\begin{array}{l}\text { Flow cytometry } \\
\text { - } \\
\text { scRNAseq }\end{array}$ & $\begin{array}{l}\text { - Immune cell specific } \\
\text { populations } \\
\text { - Transcriptomes/genetic } \\
\text { signatures }\end{array}$ & {$[119],[120]$} \\
\hline & Renal function & $\begin{array}{l}\text { - FITC-insulin clearance } \\
\text { - Albumin excretion rate } \\
\text { - Serum creatinine levels }\end{array}$ & $\begin{array}{l}\text { - Alteration in glomerular } \\
\text { filtration rate } \\
\text { - Degree of renal dysfunction }\end{array}$ & [110] \\
\hline & $\begin{array}{l}\text { Inflammatory and oxidative } \\
\text { stress markers }\end{array}$ & $\begin{array}{ll}\text {. } & \text { Western Blot } \\
\text {. } & \text { IHC/IF } \\
\text {. } & \text { ELISA } \\
\text {. } & \text { PCR }\end{array}$ & $\begin{array}{l}\text { Renal structural alterations } \\
\text { Specific antibodies can be } \\
\text { utilised to investigate protein } \\
\text { and gene expression of } \\
\text { inflammatory and oxidative } \\
\text { markers }\end{array}$ & [107-115] \\
\hline & $\begin{array}{l}\text { Systemic distribution after } \\
\text { injection }\end{array}$ & - Optical fluorescence imaging & $\begin{array}{l}\text { - In vivo/ex vivo biodistribution } \\
\text { of fluorochrome-conjugated } \\
\text { drug }\end{array}$ & [114] \\
\hline \multirow{5}{*}{ 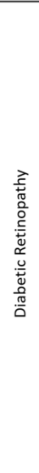 } & Retinal function & - Electroretinography & - Retinal function alteration & {$[26],[128],[129]$} \\
\hline & Retinal vasculature & $\begin{array}{l}\text { - Laser speckle flowgraphy } \\
\text { - Fundoscopy and fluorescein } \\
\text { angiograms } \\
\text { - Optical coherence } \\
\text { tomography imaging }\end{array}$ & $\begin{array}{l}\text { Degree of blood flow volume, } \\
\text { ocular perfusion and } \\
\text { dysregulated retinal } \\
\text { microvasculature } \\
\text { Vascular leakage, vessel } \\
\text { dilation and beading } \\
\text { - Retinal hyper-reflective foci, } \\
\text { sub-retinal fluid severity, } \\
\text { retinal layer thickness }\end{array}$ & {$[26],[126-129]$} \\
\hline & $\begin{array}{l}\text { Inflammatory and oxidative } \\
\text { stress markers }\end{array}$ & $\begin{array}{ll}\text {. } & \text { Western Blot } \\
\dot{0} & \text { IHC/IF } \\
\text {. } & \text { ELISA } \\
\text {. } & \text { PCR }\end{array}$ & $\begin{array}{l}\text { Specific antibodies can be utilised } \\
\text { to investigate protein and gene } \\
\text { expression of inflammatory and } \\
\text { oxidative markers }\end{array}$ & {$[26],[128],[129]$} \\
\hline & $\begin{array}{l}\text { Leucocyte mediated endothelial } \\
\text { cell death }\end{array}$ & - Flow cytometry & $\begin{array}{l}\text { - Degree of retinal endothelial } \\
\text { cell death }\end{array}$ & [26] \\
\hline & VMAT2 & - $\quad\left[{ }^{18} \mathrm{~F}\right] F P-(+)-D T B Z$ PET imaging & - Loss of dopaminergic neuron & [129] \\
\hline \multirow{4}{*}{ 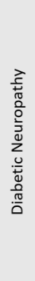 } & Nerve conduction & $\begin{array}{l}\text { - Nerve conduction velocity } \\
\text { test }\end{array}$ & $\begin{array}{l}\text { - Alteration in nerve } \\
\text { conduction velocity }\end{array}$ & [134-135] \\
\hline & $\begin{array}{l}\text { Sensory and nerve } \\
\text { electrophysiological impairments }\end{array}$ & - Pain assessment & $\begin{array}{l}\text { Withdrawal response to } \\
\text { thermal and mechanical } \\
\text { stimulation }\end{array}$ & {$[135],[137],[138]$} \\
\hline & $\begin{array}{l}\text { Intraepidermal nerve fibre } \\
\text { density }\end{array}$ & $\begin{array}{l}\text { - Protein gene product } 9.5 \\
\text { Immunofluorescence }\end{array}$ & $\begin{array}{l}\text { - Morphological and functional } \\
\text { changes of small-fibres } \\
\text { - Functional innervations of } \\
\text { the skin }\end{array}$ & {$[134],[135],[138$} \\
\hline & $\begin{array}{l}\text { Morphological nerve } \\
\text { regeneration }\end{array}$ & $\begin{array}{l}\text { - Fluorescence } \\
\text { stereomicroscopy }\end{array}$ & $\begin{array}{l}\text { - Tracking regeneration of } \\
\text { proper plantar digital nerves } \\
\text { in thy1-YFP transgenic mice }\end{array}$ & [139] \\
\hline
\end{tabular}

Table 2: Summary of experimental analysis and research output for models of diabetes-associated microvascular complications. 